\title{
Sorafenib Is Effective in the Treatment of Pulmonary Veno-Occlusive Disease
}

\author{
Masaharu Kataoka ${ }^{a}$ b Ryoji Yanagisawa $^{a}$ Keiichi Fukuda ${ }^{b}$ Hideaki Yoshino $^{a}$ \\ Toru Satoha

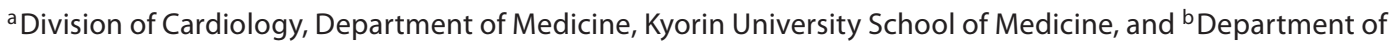 \\ Cardiology, Keio University School of Medicine, Tokyo, Japan
}

\section{Established Facts}

- The efficacy of sorafenib, a multikinase inhibitor, in the treatment of pulmonary arterial hypertension $(\mathrm{PAH})$ has been explored and, indeed, studies have demonstrated that it improves experimental $\mathrm{PAH}$ in animals.

- Pulmonary veno-occlusive disease (PVOD) is a disease in which the lesions affect the pulmonary capillary veins and not the pulmonary arteries. The efficacy of sorafenib in the treatment of pulmonary hypertension other than PAH, such as PVOD, is still unclear.

- Combination therapy of epoprostenol plus imatinib, a tyrosine kinase inhibitor, was reported to be effective in treating a patient with PVOD.

\section{Novel Insights}

- In our case report, imatinib proved ineffective, but sorafenib improved the hemodynamics and symptoms of PVOD. Sorafenib alone is effective in the treatment of PVOD without the need for combination therapy with epoprostenol. Sorafenib may be a potential therapeutic strategy for the treatment of PVOD.

\section{Key Words}

Hypertension • Imatinib - Pulmonary veno-occlusive disease $\cdot$ Side effect $\cdot$ Sorafenib

\begin{abstract}
The present study is the first report of the effectiveness of sorafenib in the treatment of pulmonary veno-occlusive disease (PVOD). A 66-year-old woman with PVOD was started on sorafenib. After 3 months of treatment with a maximum dosage of $400 \mathrm{mg} /$ day sorafenib, there was an improvement
\end{abstract}

\section{KARGER}

Fax +41613061234 E-Mail karger@karger.ch www.karger.com (c) 2012 S. Karger AG, Basel $0008-6312 / 12 / 1233-0172 \$ 38.00 / 0$

Accessible online at:

www.karger.com/crd in the patient's New York Heart Association (NYHA) functional class from IV to III. However, because of severe painful eruptions as a side effect of sorafenib, the patient stopped sorafenib and was started on imatinib instead. This treatment resulted in a worsening of the patient's NYHA class from III to IV, so sorafenib was restarted at a reduced dosage of $300 \mathrm{mg} /$ day. The resumption of sorafenib was associated with clinical improvement, specifically NYHA class from IV to II and hemodynamic amelioration, and tolerable eruptions. In conclusion, sorafenib may be a potential therapeutic strategy for the treatment of PVOD.

Copyright $\odot 2012$ S. Karger AG, Basel

Toru Satoh, MD

Division of Cardiology, Department of Medicine

Kyorin University School of Medicine

6-20-2 Shinkawa, Mitaka, Tokyo 181-8611 (Japan)

E-Mail tsatoh@ks.kyorin-u.ac.jp 


\section{Introduction}

Sorafenib, a multikinase inhibitor, is a recently developed molecular targeting agent that has been used in the treatment of cancer [1]. The possibility of using sorafenib in the treatment of pulmonary arterial hypertension (PAH) has also been explored and, indeed, studies have demonstrated that it improves experimental $\mathrm{PAH}$ in animals $[2,3]$. Furthermore, a previous report presented the results of a phase Ib study of sorafenib in $\mathrm{PAH}$ patients [4]. However, the efficacy of sorafenib in the treatment of pulmonary hypertension other than PAH is not known.

Patients with pulmonary veno-occlusive disease (PVOD), in which the lesions affect the pulmonary capillary veins and not the pulmonary arteries, develop pulmonary hypertension, leading to right heart failure and a grave prognosis. In the present study, we report on the efficacy of sorafenib in the treatment of a patient with PVOD.

\section{Case Description}

A 66-year-old woman was diagnosed with PVOD after collagen vascular disease, pulmonary disease, pulmonary thromboembolism, left heart abnormality, and other systemic diseases had been ruled out. The diagnosis was confirmed by lung computed tomography findings compatible with PVOD. The patient was started on sorafenib at a dose of $100 \mathrm{mg} / \mathrm{day}$, which was increased to $200 \mathrm{mg} /$ day after 3 weeks, then to $300 \mathrm{mg} /$ day after another 2 weeks, and finally to $400 \mathrm{mg} /$ day after 2 weeks, dose at which it was maintained.

The study protocol was approved by the Ethics Committee at Kyorin University Hospital. The purpose of the study was explained to the patient, who provided written informed consent before sorafenib treatment was started.

Figure 1 shows the time course of changes in the patient's New York Heart Association (NYHA) functional classification. Three months after starting sorafenib, the patient's NYHA functional classification had improved from class IV to class III. However, as a side effect of sorafenib, the patient developed mildly swollen eruptions that were itchy and painful. Thus, sorafenib treatment was stopped.

As an alternative to sorafenib, the patient was started on 200 $\mathrm{mg} /$ day imatinib, a tyrosine kinase inhibitor. After cessation of sorafenib treatment, the eruptions improved. However, after 1 month of treatment with imatinib, the patient's NYHA functional classification had deteriorated to nearly class IV, her symptoms and dyspnea were exacerbated, and leg edema developed due to right-sided heart overload. Thus, imatinib treatment was deemed ineffective for this patient and was stopped.

The patient was restarted on sorafenib at a dose of $300 \mathrm{mg} /$ day. After 7 months with $300 \mathrm{mg} /$ day sorafenib, the patient's NYHA classification had improved to class II. Although the eruptions also redeveloped as a side effect of sorafenib, they were minor and tolerable.

The right-sided heart catheterization and 6-min walk distance (6MWD) tests were performed at baseline and then again after 12

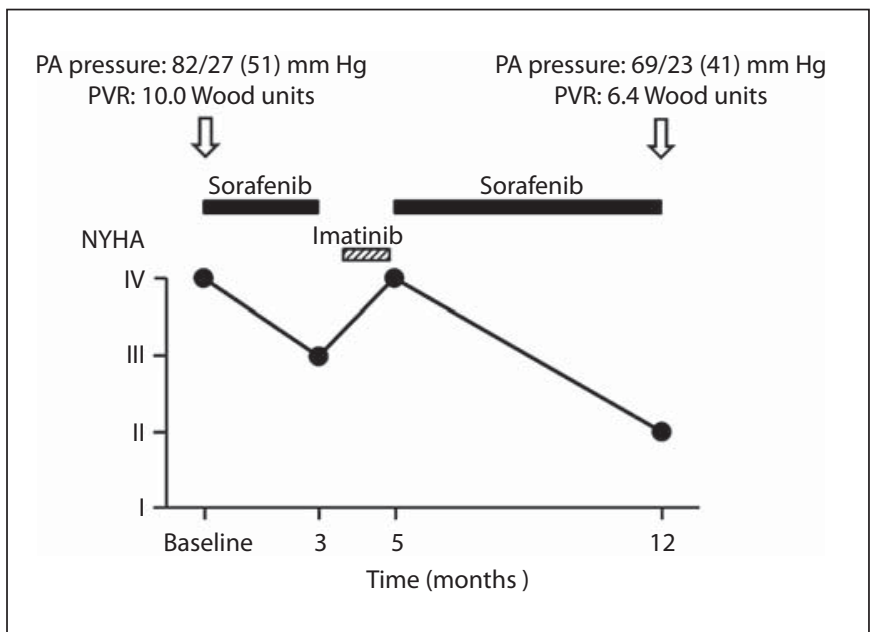

Fig. 1. Time course of changes in the patient's NYHA functional classification, pulmonary arterial (PA) pressure, and pulmonary vascular resistance (PVR) in relation to the administration of sorafenib and imatinib. A right-sided heart catheterization was performed at baseline and then again after 12 months.

months. Catheterization demonstrated improvements in pulmonary vascular resistance (from 10.0 to 6.4 Wood units), systolic pulmonary arterial pressure (from 82 to $69 \mathrm{~mm} \mathrm{Hg}$ ), mean pulmonary arterial pressure (from 51 to $41 \mathrm{~mm} \mathrm{Hg}$ ), and mean right atrial pressure (from 6 to $2 \mathrm{~mm} \mathrm{Hg}$ ), as well as an increase in cardiac output (from 4.4 to 6.1 liter/min). The 6MWD increased from 200 to $245 \mathrm{~m}$.

\section{Discussion}

In our patient, sorafenib improved the hemodynamics and symptoms of PVOD, whereas imatinib proved ineffective. In a previous case report, a combination of epoprostenol plus imatinib was reported to be effective in treating a patient with PVOD [5]; however, on the basis of the findings of the present study, it is possible that imatinib alone, without epoprostenol, would not have been sufficient to treat PVOD in the previous study. We did not use epoprostenol in our patient. Our findings suggest that sorafenib alone is effective in the treatment of PVOD without the need for combination therapy with epoprostenol. Thus, sorafenib may be a potential therapeutic strategy for the treatment of PVOD.

\section{Conflict of Interest}

None. 


\section{References}

1 Iyer R, Fetterly G, Lugade A, Thanavala Y: Sorafenib: a clinical and pharmacologic review. Expert Opin Pharmacother 2010;11: 1943-1955.

-2 Klein M, Schermuly RT, Ellinghaus P, Milting H, Riedl B, Nikolova S, Pullamsetti SS, Weissmann N, Dony E, Savai R, Ghofrani HA, Grimminger F, Busch AE, Schäfer S: Combined tyrosine and serine/threonine kinase inhibition by sorafenib prevents progression of experimental pulmonary hypertension and myocardial remodeling. Circulation 2008;118:2081-2090.
Moreno-Vinasco L, Gomberg-Maitland M, Maitland ML, Desai AA, Singleton PA, Sammani S, Sam L, Liu Y, Husain AN, Lang RM, Ratain MJ, Lussier YA, Garcia JG: Genomic assessment of a multikinase inhibitor, sorafenib, in a rodent model of pulmonary hypertension. Physiol Genomics 2008;33: 278-291.
4 Gomberg-Maitland M, Maitland ML, Barst RJ, Sugeng L, Coslet S, Perrino TJ, Bond L, Lacouture ME, Archer SL, Ratain MJ: A dosing/cross-development study of the multikinase inhibitor sorafenib in patients with pulmonary arterial hypertension. Clin Pharmacol Ther 2010;87:303-310.

5 Overbeek MJ, van Nieuw Amerongen GP, Boonstra A, Smit EF, Vonk-Noordegraaf A: Possible role of imatinib in clinical pulmonary veno-occlusive disease. Eur Respir J 2008;32:232-235 